\title{
Evaluation of covalent bond density in molecular crystals using simplified virtual scattering centers: inorganic and complex compounds
}

\author{
A Nazarenko ${ }^{1}$ \\ ${ }^{1}$ Chemistry Dept, SUNY Buffalo State, Buffalo, NY \\ nazareay@buffalostate.edu
}

The Independent Atom Model (IAM) provides a good estimate for the most of routine crystallographic research. A charge density approach, which additionally describes bonding electrons, requires very high quality data in order to obtain meaningful results. Here we address a 'gray area' of good quality datasets with $0.5-0.8 \AA$ resolution, which show visible deviation from IAM but are not satisfactory for experimental charge density calculations. The suggested methodology follows the well-known virtual atom method (see [1-3] and numerous references therein. Most probably, the idea of placing a scattering center exactly in the middle of two carbon atoms was first implemented by Rosalind Franklin [1] and was 'conveniently forgotten' by all subsequent publications in this field. Virtual scattering centers (VSC) are placed at fixed calculated positions between $\mathrm{C}, \mathrm{N}$, and $\mathrm{O}$ atoms with 'occupancies' being different for single, double, aromatic, and triple bonds. Scattering is approximated by a single Gaussian which can be justified by a small value of correction. VSCs are treated as isotropic: multiplication of point VSC by Debye-Waller factor yields a single isotropic Gaussian function to describe both effects (no deconvolution of vibrations and charge density). All scattering at VSC is described as anomalous scattering; therefore, there is no change of total number of electrons in a molecule. Usual IAM software (SHELXL or Jana) was employed. The number of introduced parameters can be as low as one (for overall occupancy of the VSC part of the structure). Introduction of VSCs makes the effect similar to the scattering of one hydrogen atom per 4-5 C-C bonds, which is substantial enough to justify the effort. Alternatively, each bond can be treated separately (number of additional parameters is roughly equal to the number of bonds). This can lead to further improvement. It is possible to handle $\mathrm{C}-\mathrm{H}$ bonds as well as lone pairs in the same fashion [4]. In this presentation, several inorganic and element-organic molecules were investigated. When it was possible, charge density calculations (MoPro [5\}) and/or HARt [6] calculations [5] were performed using the same experimental data. Visible improvement of fitting characteristics was achieved, especially for molecules with aromatic fragments. Some representative examples: Sulfamic acid resolution $0.37 \AA$ : IAM R=2.1, VSC 1.86. Refinement with HARt [6] gives $\mathrm{R}=1.85$. All S-O bonds and S-N bond have approximately the same covalent scattering, in agreement with charge density results (MoPro), confirming single covalent bonds in this molecule (plus additional electrostatic interactions). Several $3 \mathrm{~d}$ transition metal complexes with large organic part and resolution of $0.58-0.62 \AA$ showed visible improvement of $\mathrm{R}$ factor (e.g, from 2.9 to 2.5 ), 30\% smaller standard deviations of bond lengths, and, more important, much cleaner residual map. After removing most of residual bond density from the Fourier difference map, other sources of deviation such as disorder and experimental and data processing limitations can be addressed. As usual, applicability of this algorithm is limited by Coppens's suitability factor S values. 1] Franklin, R. E. Nature 1950, 165, 71-72. [2] Scheringer, C. \&. Kutoglu, A. Acta Cryst. 1983, A39, 899-901. [3] Afonine, P.; Pichon-Pesme, V; Muzet, N.; Lecomte, C.; Urzhumtsev, A. CCP4 Newsletter on Protein Crystallography, 2002, 41; Afonine, P.V; GrosseKunstleve, R.W.; Adams, P D.; Lunin, V. \& Urzhumtsev, A. Acta Cryst. 2007, D63, 1194-1197; Dadda, N.; Nassour, A.; Guillot, B.; Benali-Cherif, N. \& Jelsch, C. Acta Cryst. 2012, A68, 452-463; Ahmed, M.; Nassour, A.; Noureen, S.; Lecomte, C. \& Jelsch, C. Acta Cryst.2016, B72, 75-86; Urzhumtsev A.G.; Lunin V.Y. Crystallography Rev., 2020, 26, 51-55. [4] Nazarenko, A.Y. Z. Kristallographie, 2018, 38, S44-S45 [5] Guillot, B. Viry, L. Guillot, R. Lecomte, C. \& Jelsch, C. (2001). J. Appl. Cryst. 34, 214-223 [6] Fugel, M.; Jayatilaka, D.; Hupf, E.; Overgaard, J.; Hathwar, V. R.; Macchi, P.; Turner, M. J.; Howard, J. A. K.; Dolomanov, O. V.; Puschmann, H.; Iversen, B. B.; Bürgi, H.-B. \& Grabowsky, S. IUCrJ 2018, 5, 32-44.

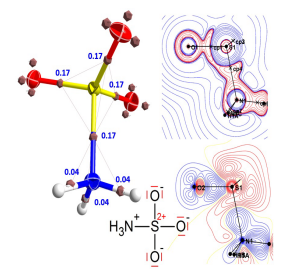

Figure 1

Acta Cryst. (2020). A76, a164 